\title{
Sibling curves and complex roots 2: looking ahead
}

\author{
Ansie Harding ${ }^{\text {a }}$ Johann Engelbrecht ${ }^{\text {a }}$ \\ a Department of Mathematics and Applied Mathematics, University of Pretoria, \\ Pretoria, South Africa
}

\section{[Figures and Tables at the bottom of the document]}

\begin{abstract}
This paper, the second of a two part article, expands on an idea that appeared in literature in the 1950s to show that by resticting the domain to those complex numbers that map onto real numbers, representations of functions other than the ones in the real plane are obtained. In other words, the well-known curves in the real plane only depict part of a bigger whole. This expanded representation brings new insight into visualising complex roots. The suggestion is that this new approach be introduced to students firstly through relating the path in history and secondly by imparting the visual presentation as exposed in the paper to offer a richer teaching and learning approach to the topic. Furthermore this approach provides a new way of employing technology to visualise concepts and curves that were previously not noticed.
\end{abstract}

\section{Background}

In the first part of this article, we traced the development of complex numbers and followed the quest for finding roots of functions, both algebraically and graphically, through millennia. The three attempts at giving visual interpretation to the existence of 
complex roots for polynomial equations, discussed in the first part of this paper, are all informative but at the same time somewhat artificial. In a first approach the Argand plane had to be superimposed onto the Cartesian plane to make the complex roots 'visible'. In the second approach the two planes were drawn next to each other and the complex roots could be traced by moving from the one plane to the other. The third approach is probably the most 'natural' of the three ways of visualising complex roots, but even here the approach is still somewhat contrived. Because it was difficult to visualize the 4dimensional graph, the modulus of the function value was taken (a real number) to eliminate the fourth dimension and make a picture of a 3-dimensional surface possible. This journey through history brought us to a point where we can expand our vision of functions and their complex roots.

\section{Working with real-valued functions}

Without much ado and seemingly almost unobserved, an idea appeared in print in 1951, in an American secondary school textbook 1. The author, Howard Fehr, is a past president of the National Council for Teachers in Mathematics in the USA and was, at the time, professor at Columbia University. The idea is elegant and under-explored, and it is the intention of this paper to expand on and run with the idea.

In short: For a function $f$ that maps complex numbers onto complex numbers, we restrict the domain to those complex numbers that map onto real values. On the new domain the function has real values and can be represented in three dimensions with the domain in the horizontal plane and the range along the vertical axis.

\footnotetext{
If the function $f$ maps complex numbers onto complex numbers then it can be written in the form

$$
y=f(z)=f(x+i w)=g(x, w)+i h(x, w)
$$

If, on the other hand, $f$ maps the complex number $x+i w$ onto the real number $y$ then $h(x, w)=0$. We restrict the domain of the function $f$ to all points in the $x w$-plane such that $h(x, w)=0$. The condition that $h(x, w)=0$ defines a curve(s) in the Argand plane (the $x w$-plane). This curve(s) acts as the domain of another curve(s) for which the formulation may be obtained by substituting the solution of $h(x, w)=0$ into equation (1). What emerges then is the realisation that there is a world beyond the real plane and that a curve in the real plane might have, what we will call, sibling curves that do not live in the real plane.
} 


\subsection{The quadratic function}

An example is appropriate at this point and we will rely on the quadratic function for this purpose. If $f(z)=A z^{2}+B z+C$ and $z$ is the complex number $x+i w$ then

$$
f(z)=\left(A x^{2}-A w^{2}+B x+C\right)+i(2 x w A+B w)
$$

For $f(z)$ to be real requires that $w=0$ or $x=-(B / 2 A)$. Thus, $f(z)$ is real in the plane $w=0$ (the Cartesian plane) and in the plane $x=-(B / 2 A)$ (perpendicular to the Cartesian plane and parallel to the $y w$-plane).

In the real plane $w=0$ and equation (2) yields

$$
f(x)=A x^{2}+B x+C, \quad x \in \mathbb{R}
$$

the well-known parabola that we have always considered as the total graph.

However, in the plane $x=-(B / 2 A)$, perpendicular to the Cartesian plane, equation (2) yields

$$
f(w)=-A^{2} w^{2}+\frac{4 A C-B^{2}}{4 A}, \quad w \in \mathbb{R}
$$

Thus the graph of $f$ consists of a parabola in each of the two perpendicular planes. If the one parabola is concave up the other is concave down and they are joined at the apexes. There are two parabolas and not only one as we traditionally have been led to believe. That the well-known parabola has a Siamese twin (or sibling curve) is an enlightening thought. The complex roots now have new meaning. They occur

at the points where the sibling curve cuts the complex plane and obviously form a conjugate pair.

As an example, consider the quadratic function $f(z)=z^{2}+2 z+2$. Apart from the function in the Cartesian plane, $y=x^{2}+2 x+2$, there is also a sibling in the plane $x=-1$, given by $y=-w^{2}+1$ and shown in figure 1 .

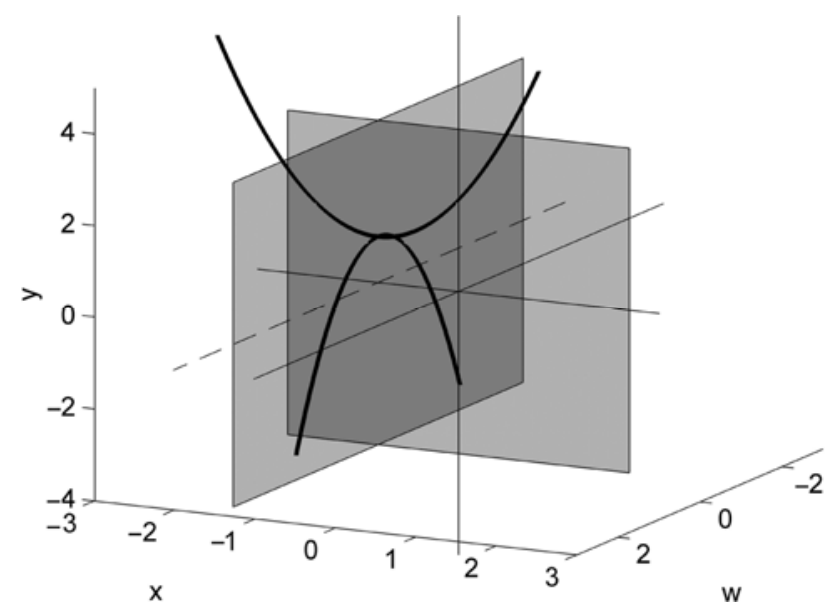

Figure 1. Quadratic function $f(z)=z^{2}+2 z+2$. 
The ideas of Fehr, exposed here, were picked up more than 30 years later by Vest 2 in 1985 and reiterated for the general case of $f(z)=A z^{2}+B z+C$, but have remained dormant since, to the best of our knowledge. In a recent article by Ballew 3, 18 ways to solve quadratic equations by analytic and graphic methods were discussed, sub-headed by 'Including those you may never have seen'. It was noticeable that although there is a passing reference to Fehr's book, this approach is absent. Another recent discussion in the Teacher2Teacher forum on the internet 4 speculates about the existence of what the enquirer refers to as phantom curves, which seem to be what we call sibling curves, but no definite conclusion was reached as to their existence.

\subsection{The cubic function}

The next obvious step in following Fehr's idea is to investigate the cubic function and trace its lesser-known siblings. The general cubic function is given by $x=z^{3}+p z+q$ (obtained through a linear transformation as mentioned in the first part of this paper).

If $z=x+i w$ then

$$
y=x^{3}-3 x w^{2}+p x+q+i w\left(3 x^{2}-w^{2}+p\right)
$$

Since we restrict ourselves to real values in the range of the function, the imaginary part must be zero, which requires that $w=0$ or $3 x^{2}-w^{2}-p=0$ (as was shown in Ward 5). The first condition gives us the well-known cubic curve in the Cartesian plane, and the second condition tells us that there are two more sibling curves, each with one of the two branches of the hyperbola $3 x^{2}-w^{2}-p=0$ as domain in the $x w$-plane. These curves therefore lie in the two hyperbolic cylinders parallel to the $y$-axis. An example is appropriate.

Consider the function $y=z^{3}+4 z+1$.

By setting ${ }^{z=x+i w}$ we can derive that, apart from the big brother curve in the Cartesian plane, there are two other siblings, defined on the hyperbolic cylinders

$$
w^{2}=3 x^{2}+4, \text { and given by } y=-8 x^{3}-8 x+1 \text {. }
$$


Figure 2(a) is a plan view showing the domain curves and figure 2(b) shows a 3-D view of the three sibling curves of $y=z^{3}-4 z+1$ on the domain curves.
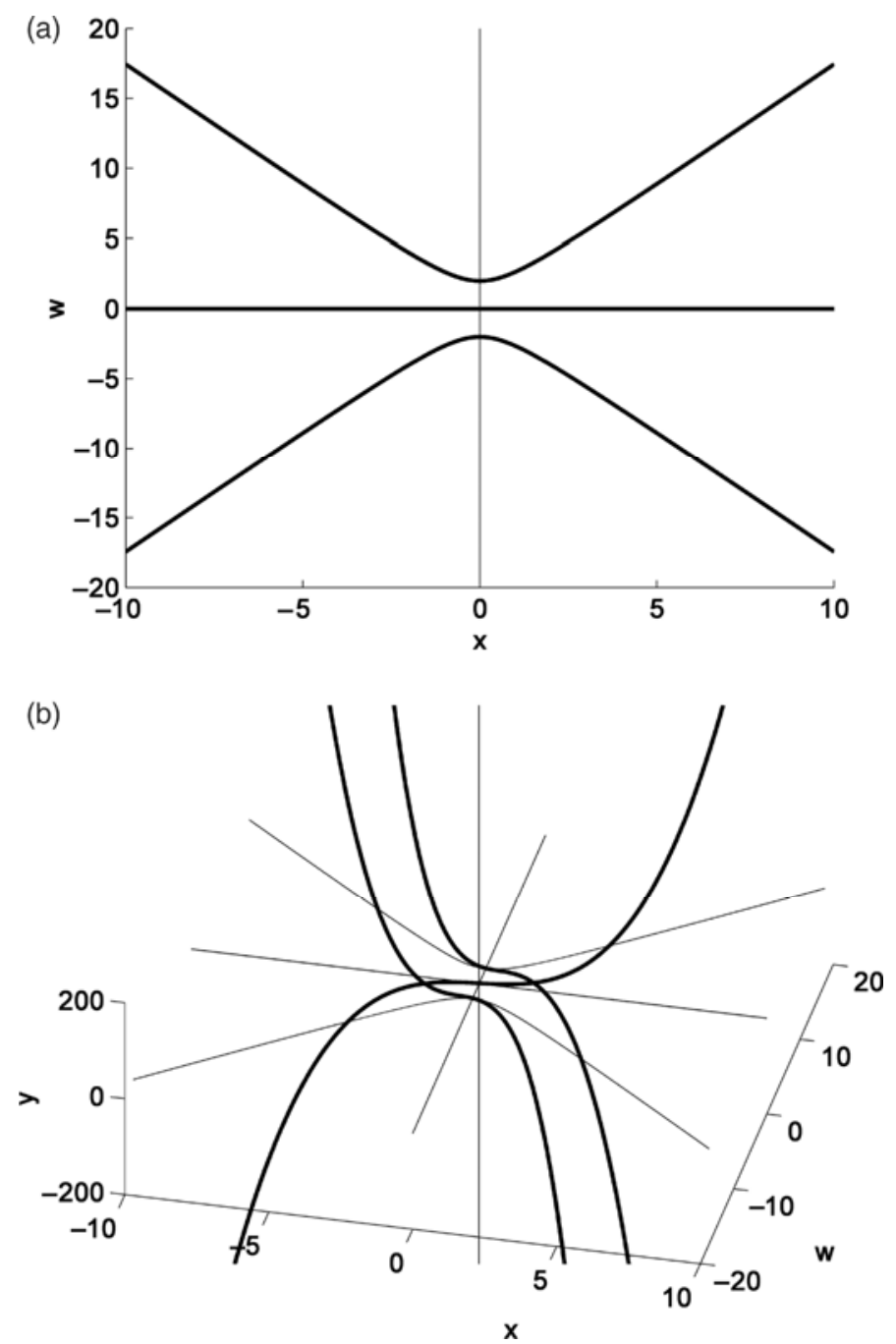

Figure 2. (a) Domain of the function $f(z)=z^{3}-4 z+1$. (b) Siblings of the function $f(z)=z^{3}-4 z+1$.

Note the following:

- The curve in the Cartesian plane, the big brother curve (bold), is increasing in the $x$-direction and the other two siblings are decreasing.

- The new curves are symmetrical about the $x y$-plane (twin curves).

- The conjugate complex roots of the twin curves are visually obvious. 
- The example illustrates curves for a positive $p$-value. For a negative $p$-value the hyperbolic domain of the new twin curves will be symmetric about the w-axis.

- Ward 5 identified the two hyperbolic domain curves (as discussed) but without identifying the sibling curves.

- There are three different cases for the domain curves of the cubic, the first with the $x$-axis as transverse axis, the second with the $w$-axis as transverse axis and the degenerate case where the domain curves are two straight lines 6.

An immediate thought that comes to mind is that if the quadratic and cubic polynomials have sibling curves, what about higher degree polynomials such as the quartic, the quintic? If the quadratic function has one sibling curve and the cubic has two, will the quartic have three? How about the other functions such as the trigonometric functions, the exponential, the circle, the hyperbola?

We will systematically go about addressing some of this host of questions.

\subsection{Quartic polynomials and beyond}

We show here that by following the same process for the quartic polynomial, there are three domain curves in the $x w$-plane onto which three more sibling curves, in addition to the big brother in the Cartesian plane, are defined. The general quartic is given by $f(g)=z^{4}+F^{2}+g z+r$ (again through a linear transformation). By setting $z=x+i w$ we obtain

$$
\begin{aligned}
f(z)= & x^{4}-6 x^{2} w^{2}+w^{4}+p x^{2}-p w^{2}+q x+r \\
& +i w\left(4 x^{3}-4 x w^{2}+2 p x+q\right)
\end{aligned}
$$

For the $i$-term to vanish, ${ }^{w=0}$ (and we get the Cartesian curve) or

$$
4 x^{3}-4 x w^{2}+2 p x+q=0
$$

which implicitly defines domain curves in the $x w$-plane. Note that $x$ cannot be zero for $q \neq 0$.

The sibling curves are obtained by substituting

$$
w^{2}=\frac{4 x^{3}+2 p x+q}{4 x}
$$


into

$$
y=x^{4}-6 x^{2} w^{2}+w^{4}+p x^{2}-p w^{2}+q x+r \mathrm{t}
$$

o obtain $y$ as a function of $x$.

We look at the example $y=z^{4}+2 z^{2}+z+2$. Following the same procedure, the big brother curve $y=x^{4}+2 x^{2}+x+2$ in the Cartesian plane is obtained. The domain curves of the other siblings are given by $4 x^{3}-4 x^{2}+m^{2}+4 x+1=0$ and the actual siblings are given by $x=x^{4}-6 x^{2} y^{3}+2 x^{4}+2 x^{2}-2 e^{2}+x+2$. Figure 3(a) shows the domain curves of all four siblings and figure 3(b) shows the four siblings on the domain curves.
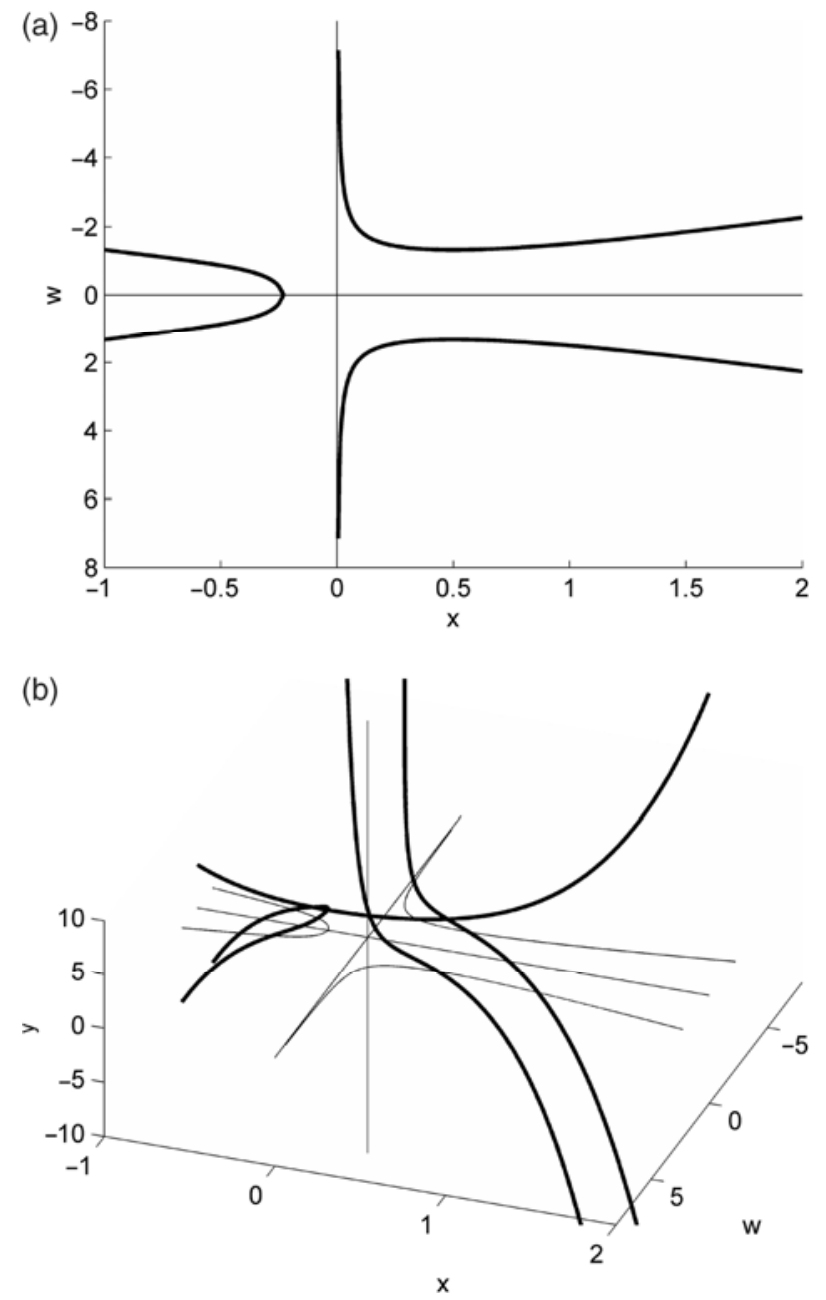

Figure 3. (a) Domain of the function $f(z)=z^{4}+2 z^{2}+z+2$. (b) Siblings of the function $f(z)=z^{4}+2 z^{2}+z+2$. 
Note the following:

- The big brother curve (bold) does not intersect the $x$-axis, so there are no real roots.

- The other three siblings do have roots and these can be graphically seen to form two conjugate pairs $(1 / 2)(1 \pm \sqrt{7} i),(1 / 2)(-1 \pm \sqrt{3} i)$.

For the quintic polynomial one is led to expect five sibling curves in total and the roots should also be visually obvious. The intention is to investigate this premise and other features of polynomial siblings in a subsequent paper.

\subsection{Exponential functions}

Having demonstrated the existence of sibling curves for polynomials, it is natural to speculate about the existence of sibling curves for the other well-known functions. Would it be possible, for example, for the exponential function to have siblings that we have not previously been aware of? We investigate.

If $f(x)=e^{x+f e x}=e^{x}(\cos w+i \sin s)$ and we are only interested in that part of the domain where $f(z)$ is real valued, then

$$
e^{x} \sin w=0 \text { which means that } w=n \pi(n \in \mathbb{Z}),
$$

so that $y=e^{x} \cos n \pi, n \in \mathbb{Z}$ The answer is positive, there are many siblings in the family.

The sibling curves of the exponential function (including the big brother curve) are given by

$$
y=\left\{\begin{array}{c}
e^{x} \text { if } n \text { is even, } w=n \pi \\
-e^{x} \text { if } n \text { is odd, } w=n \pi
\end{array}\right\}
$$

and shown in figure 4. 


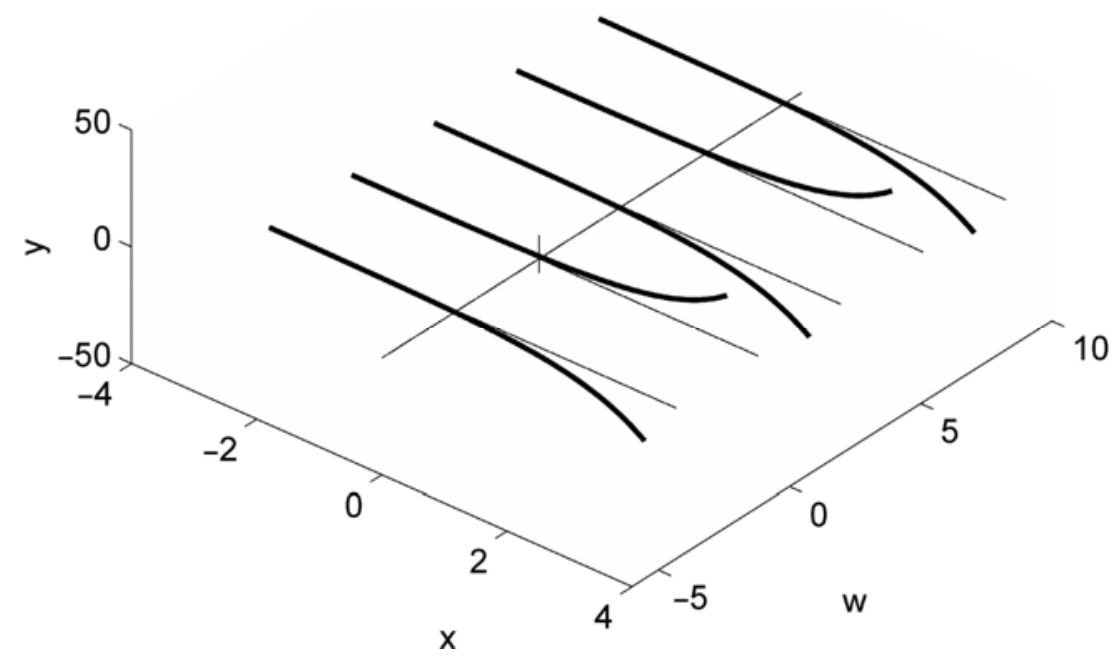

Figure 4. Siblings of the exponential function.

The understanding is that the exponential function as we know it in the Cartesian plane is just one of infinitely many siblings, evenly spaced along the $w$-axis and reversing sign every $\pi$-units. None of the sibling curves of the exponential function ever cuts the $x w$ plane, so there are no roots, real or complex.

\subsection{Trigonometric and hyperbolic functions}

We know the cos function as a periodic function in the Cartesian plane that has roots every $\pi$ units. We also know that the cos function has real roots only but is it possible that it still has sibling curves and if so, would these also be trigonometric functions?

If $f(z)=\cos z=\cos (x+i \omega)$ then

$$
f(z)=\cos x \cosh w+i \sin x \sinh w
$$

If, once again, we only consider that part of the domain of the function $f$ for which $f(z)$ is real valued, then $\sin x \sinh w=0$.

So either $\sin x=0$ which happens when $x=n \pi(n \in \mathbb{Z})$ or $\sinh w=0$ which happens only when $w=0$. The latter case gives the normal Cartesian curve. For the first case, it appears that there are sibling curves in the planes $x=n \pi$ ( $N \in \mathbb{*}$ ). These curves are obtained by substituting $x=n \pi$ into equation (3):

$$
y=\cos x \cosh y=\left\{\begin{array}{c}
\cosh w \text { if } n \text { even, } x=n \pi \\
-\cosh w \text { if } n \text { odd, } x=n \pi
\end{array}\right\}
$$


We now realize that the cos function is part of a much larger family. The cos function has infinitely many siblings, all joined to the function at its turning points. From the visual representation in figure 5 we see why the cos function has only real roots, the other sibling curves do not have any roots. We also see that the siblings of the cos function are not trigonometric functions, but hyperbolic functions.

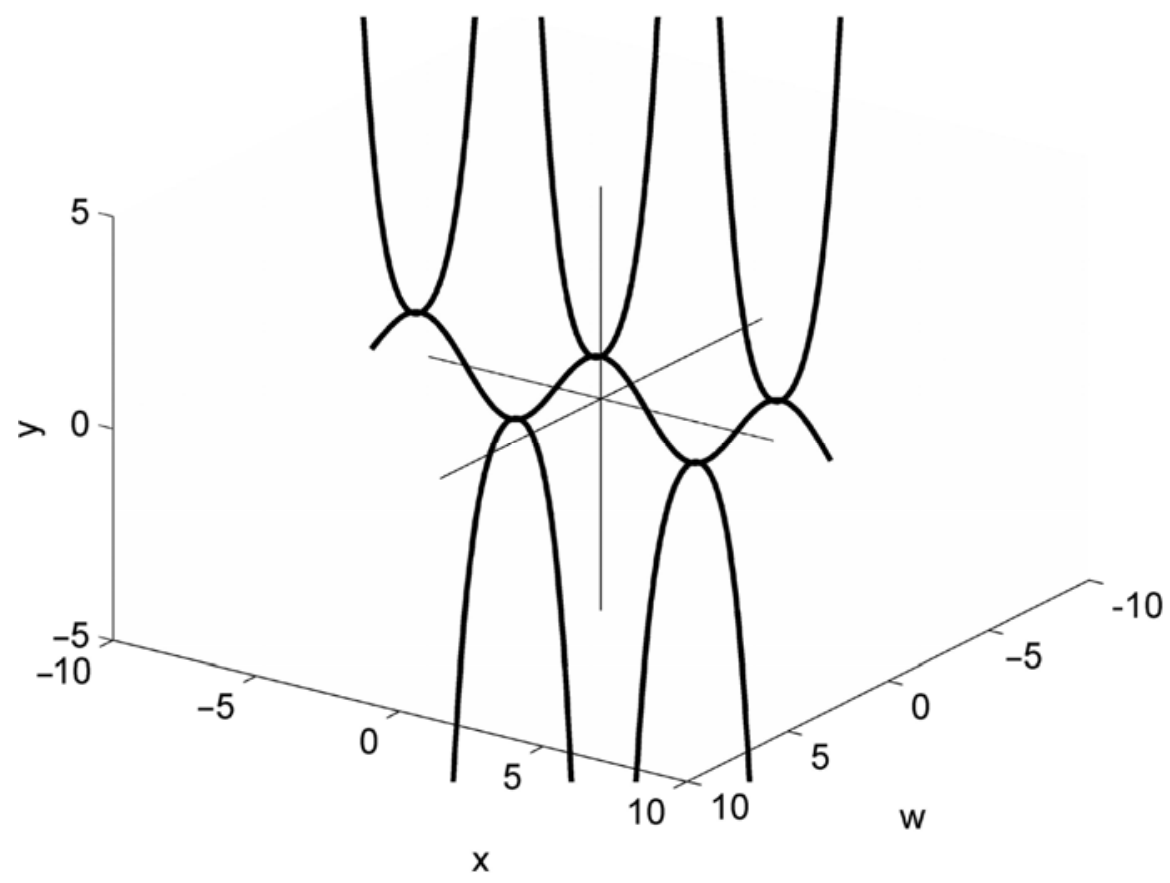

Figure 5. Function $f(z)=\cos z$.

The observed alliance between the cos function and the cosh function leads to the question whether, if we had started with the cosh function, would we have obtained the cos function as its sibling?

If

$$
f(z)=\cosh z=\cosh (x+i w) \quad\left(=\frac{e^{(x+i w)}+e^{-(x+i w)}}{2}\right)
$$

it follows that $f(c)=c \cos x \cosh x+i \sin u \sinh x$. For the function to be real valued either $\sin w=0$ for which $w=n \mathbb{R}, \mathbb{m} \in \mathbb{E}$ rendering

$$
y=\left\{\begin{array}{cc}
\cosh x, n \text { even, } \quad w=n \pi \\
-\cosh x, n \text { odd, } \quad w=n \pi
\end{array}\right\}
$$

or $\sinh x=0$ for which $x=0$ and which renders $y=\cos w$. So for the cosh function in the Cartesian plane there is a cos sibling in the $y w$-plane and many other cosh siblings running parallel to the big brother. 
The answer to the question is yes, the cosh function does indeed have the cos function as one of its siblings and the alliance holds both ways. But there are other siblings of the cosh function too, it repeats itself and changes sign every $\pi$ units on the $w$-axis.

It can be shown that the sin-function has sibling curves when $x=(n z / 2)(n \in \mathbb{Z})$, that is at the turning points of the function with the sibling curves given by

$$
y=\left\{\begin{array}{c}
\cosh x, n=4 k+1, k \in \mathbb{Z}, w=\frac{n \pi}{2} \\
-\cosh x, n=4 k-1, k \in \mathbb{Z}, w=\frac{n \pi}{2}
\end{array}\right\}
$$

Another surprising result is that the siblings of sinh-function behave similarly to those of the exponential function $f(z)=e^{z}$. The sinh function repeats itself and changes sign every $\pi$-units on the $w$-axis. This means that the sinh-function does not only have a root at zero, it has complex roots at $z=i \mathrm{H} \pi(k \in \mathbb{Z})$.

\subsection{The circle}

The circle is known to have only real roots but as we now know that does not exclude itself from having sibling curves. We investigate the possibility.

If

$$
y^{2}+z^{2}=r^{2} \quad \text { or } y^{2}+(x+i w)^{2}=r^{2} \text { then } y^{2}=r^{2}-x^{2}+w^{2}-2 i x w
$$

For $y$ to be real it is necessary and sufficient that $y^{2}$ be real and positive. The coefficient of $i$ must be zero, so

$$
x=0 \quad \text { or } \quad w=0
$$

For $w=0$ we obtain the circle in the Cartesian plane $y^{2}+x^{2}=r^{2}$ and for $x=0$ we obtain the sibling curve $y^{2}-w^{2}=r^{2}$ which is a hyperbola in the $y w$-plane with the $y$-axis as transverse axis.

The circle not only has sibling curves but, once again, there is an unexpected alliance with another, seemingly unrelated, function, the hyperbola. Figure 6 shows a circle and its two perpendicular sibling curves which together form a hyperbola. 


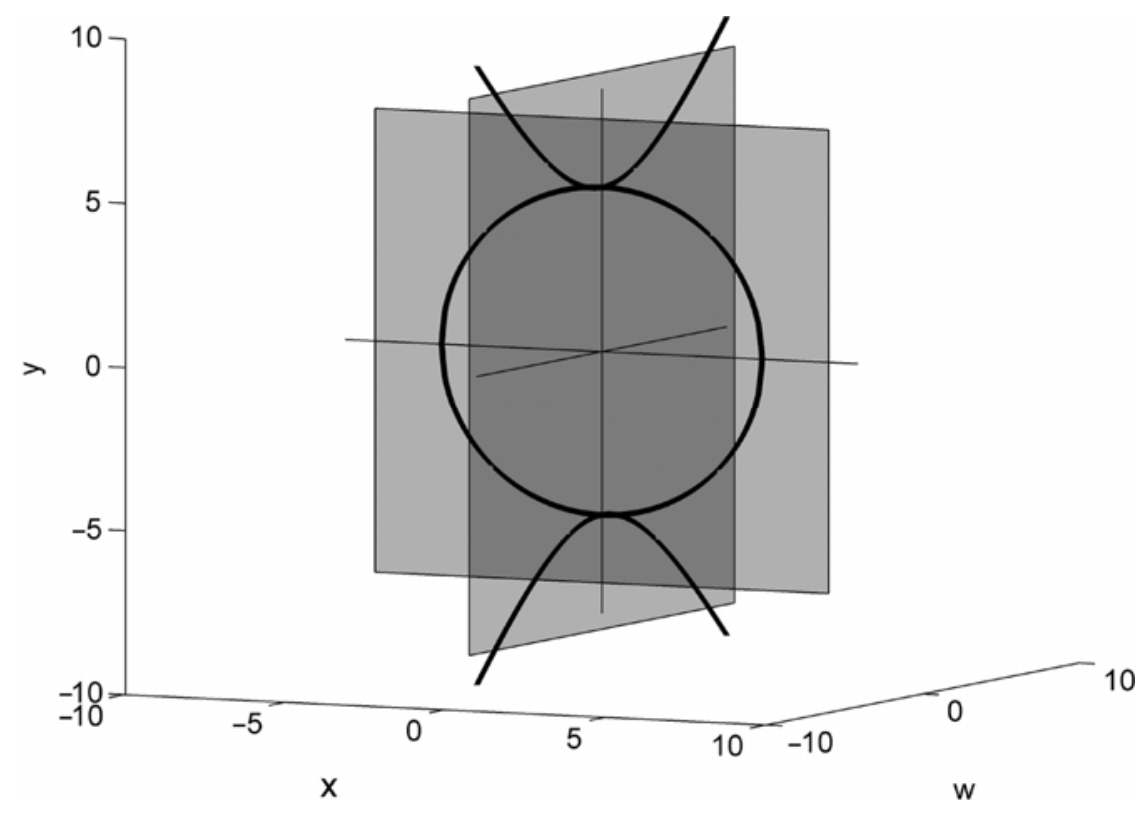

Figure 6. Function $y^{2}+z^{2}=25$.

The alliance between the circle and the hyperbola, of course, leads to the question whether the alliance holds both ways. In other words, had we started with a hyperbola in the Cartesian plane, would it similarly produce a circle as a sibling?

This is indeed the case as can be verified. However, it is only the hyperbola with the $x$ axis as transverse axis that has the circle as sibling, the 'vertical' hyperbola has no siblings.

Finally, we give new insight into a familiar topic. 


\subsection{Solutions of $z^{n}=1$}

De Moivre's theorem is standard to the content of any introductory course in complex numbers and in any such course students come to learn that the $n$ complex roots of $f(z)=z^{n}-1$ lie on a circle in the Argand plane and are evenly spaced. We will try to give a new graphical representation of these complex roots by tracing the sibling curves of this function.

We start by looking at the function $y=f(g)=z^{8}-1=(x+i \varepsilon)^{3}-1$ which renders

$$
y=x^{3}-3 x w^{2}-1+i\left(3 x^{2} w-w^{3}\right)
$$

For $f$ to be real valued, either $w=0$ or $3 x^{2}-w^{2}=0$. The first condition gives the Cartesian curve $y=x^{3}-1$. The second condition tells us that the domain of the sibling curves consists of two straight lines $w= \pm \sqrt{3} x$ Substituting $w^{2}=3 x^{2}$ into (4) gives the function $y=-8 x^{3}-1$ defined on the two lines and shown in the figure 7.

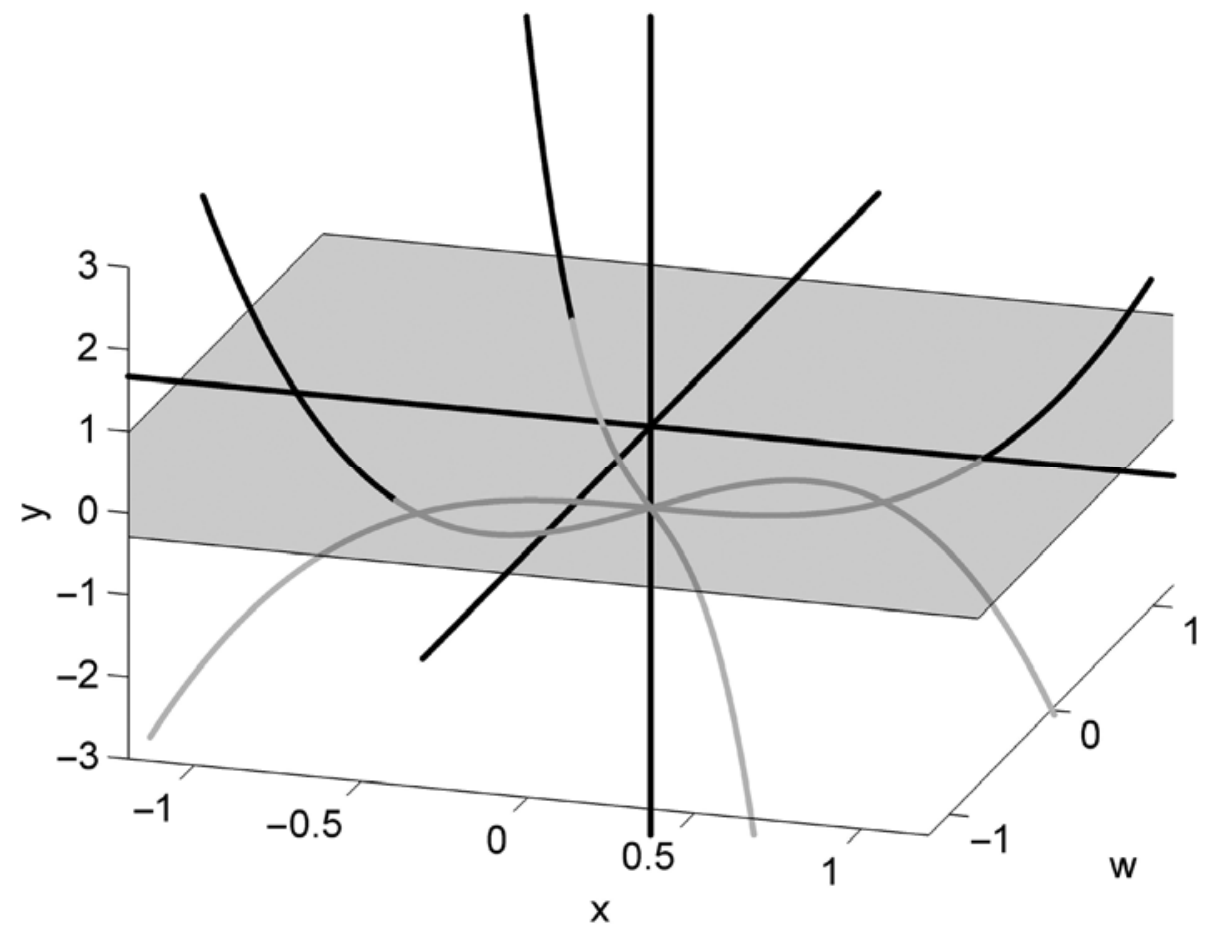

Figure 7. Function $f(z)=z^{3}-1$. 
Notice that the Cartesian curve is accompanied by two siblings, all of them intersecting the $y$-axis at -1 and intersecting the $x w$-plane at the real root 1 and the two complex roots $(1 / 2) \pm /(\sqrt{3} / 2)$. The three siblings curves come together like a bunch of flowers at -1 on the $y$-axis.

The picture becomes even more spectacular when $n$ has a bigger value. For example, for $n=6$ there will be six siblings, altogether, all intersecting the $y$-axis at -1 and symmetrically spaced around this axis as shown in figure 8 . The bunch of flowers becomes bigger as $n$ increases. This illustration of the roots of $z^{n}-1$ is both mathematically and aesthetically pleasing.

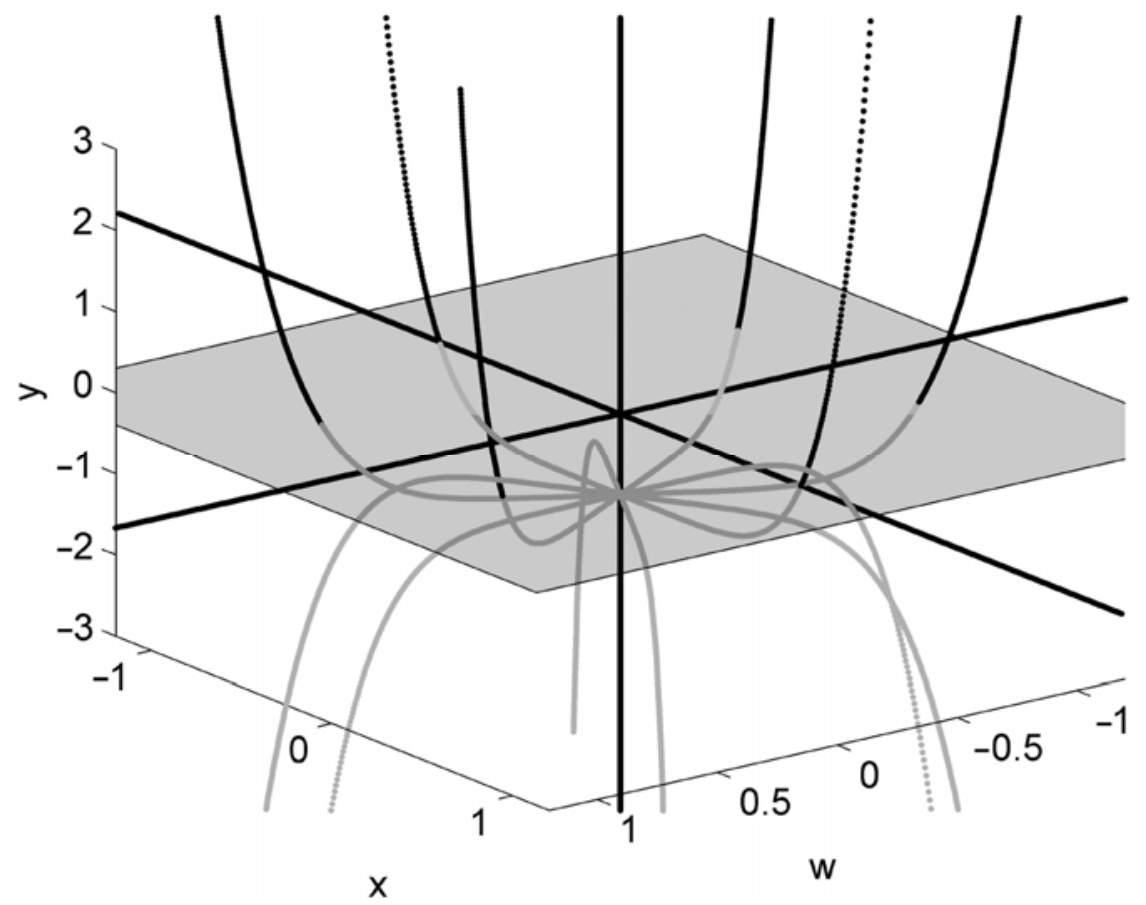

Figure 8. Function $f(z)=z^{6}-1$. 


\section{In conclusion}

Graphical function and root representation has developed over centuries, from primitive calculations to formulae in closed form to the multiple of possibilities of the technology era. The approach outlined here gives a fresh view to graphical representation of complex roots and general understanding of the functions that we deal with every day. It opens up the world of functions and, once aware of the existence of the so-called sibling curves, it is hard to look at functions in their previously perceived solitary way. This visual interpretation of complex roots, unlike the other attempts discussed in the first part of the paper, is the natural way of representing these roots visually. This approach, especially for comprehending the Fundamental theorem of Algebra and the positioning of complex roots is not only recommended, it is deemed as essential for teaching and learning. This exposition lifts the visualisation of complex roots into the new technology era. Not only is technology used for visualisation of complex roots but it enables us to have a more comprehensive view of the well-known functions.

\section{References}

- 1. Fehr, H. (1951) Secondary Mathematics Heath, Washington, DC

- 2. Vest, F. (1985) Graphing the complex roots of a quadratic equation. The College Mathematics Journal 16:4 , pp. 257-261.

- 3. Ballew, P. Solving quadratic equations by analytic and graphic methods; including several methods you may never have seen. Unpublished article Retrieved April 2007 from http://www.pballew.net/quadsol.doc

- 4. Teacher2Teacher (2003) Internet discussion on Mathforum. The geometry of the complex roots of graphs - Retrieved April 2007 from http://mathforum.org/t2t/message.taco?thread $=12605 \&$ message $=1$

- 5. Ward, JA (1937) Graphical representation of complex roots. National Mathematics Magazine 11:7, pp. 297-303.

- 6. Renka, RJ and Vest, F. (1988) Graphical representation of complex functions. Mathematics and Computer Education 22:1 , pp. 33-45. 